\title{
A GAS PROPORTIONAL CHAMBER FOR USE IN COSMIC X-RAY RESEARCH
}

\author{
P. SERLEMITSOS
}

Goddard Space Flight Center, Greenbelt, Md.. U.S.A.

\begin{abstract}
Multi-anode gas proportional counters, when used in cosmic X-ray research, offer several advantages over more conventional large area detectors. Some of these are:

1. Low detector background, effected by excluding from the sensitive volume, areas in the proximity of the counter walls, and by requiring that the region of interaction is localized inside the counter.

2. Uniform response which makes possible energy resolution limited primarily by the statistics on the number of electron-ion pairs in the interaction, rather than by partial charge collection due to weak field regions inside the counter.

3. Information relating to the interaction depth within the gas, which may be used in applying consistency checks regarding the nature of the incident radiation.

Methods have been devised for the modular construction of such counters, with particular emphasis on such items as, large areas, ease of assembly, matching of the many counter modules, accurate collimation, window supports, and shape integrity under differing amounts of differential pressure. Detector background at rocket altitudes was found to be flat above $2 \mathrm{keV}$, and less than $10^{-3} \mathrm{~cm}^{-2} \mathrm{~s}^{-1} \mathrm{keV}^{-1}$.
\end{abstract}

\section{DISCUSSION}

H. F. lan Beek: What is the reason for having so much wires between the different sections of the counter? What is the dynamic range of the instrument?

$P$. Serlemitsos: The large number of ground wires used was necessary to reduce the cross talk between cells. The dynamic range is $260 \mathrm{keV}$. 